\title{
A New Technique to Induce Experimental Myointimal Hyperplasia
}

\author{
Sami Asfar ${ }^{a}$ Ali Shuaib $^{b}$ Fatemah Al-Otaibi $^{a}$ Sora S. Asfar ${ }^{c}$ \\ Narayana Kilarkajed \\ ${ }^{a}$ Department of Surgery, Faculty of Medicine, Kuwait University, Kuwait City, Kuwait; ${ }^{b}$ Biomedical Engineering \\ Unit, Department of Physiology, Faculty of Medicine, Kuwait University, Kuwait City, Kuwait; ' ${ }^{\circ}$ epartment of \\ Microbiology, Faculty of Medicine, Kuwait University, Kuwait City, Kuwait; ${ }^{d}$ Department of Anatomy, Faculty of \\ Medicine, Kuwait University, Kuwait City, Kuwait
}

\section{Significance of the Study}

- In this paper, we describe a new innovative technique to induce experimental myointimal hyperplasia using a dental flossing cachet. The technique is simple, inexpensive, and highly reproducible. It would be of interest to researchers studying the pathogenesis of myointimal hyperplasia.

\section{Keywords}

Angioplasty $\cdot$ Carotid stenosis · Intimal injury $\cdot$ Myointimal hyperplasia $\cdot$ Neointima formation

\begin{abstract}
Background: Arterial myointimal hyperplasia $(\mathrm{MIH})$ has a significant impact on the long-term outcomes of vascular procedures such as bypass surgery and angioplasty. In this study, we describe a new and innovative technique to induce MIH using a dental flossing cachet in Wistar rats. Methods: The intimal damage in the common carotid artery was induced by inserting the tip of the dental flossing cachet through the external carotid artery into the common carotid artery and turning it on for 3 rounds of 20 s each $(n=10)$. After 2 weeks, the rats were anesthetized and the common carotid arteries of the experimental side and the contralateral side (control) were harvested and preserved for histopatho-
\end{abstract}

\begin{tabular}{ll}
\hline KARGER & $\begin{array}{l}\text { (C) } 2018 \text { The Author(s) } \\
\text { Published by S. Karger AG, Basel }\end{array}$ \\
E-Mail karger@karger.com & $\begin{array}{l}\text { This is an Open Access article licensed under the Creative Commons } \\
\text { Attribution-NonCommercial-4.0 International License (CC BY-NC) } \\
\text { (http://www.karger.com/Services/OpenAccessicense), applicable to } \\
\text { the online version of the article only. Usage and distribution for } \\
\text { commercial purposes requires written permission. }\end{array}$
\end{tabular}

logical studies. Results: The experimental carotid arteries showed significant intimal proliferation and thickening compared to the controls. The intima/media ratio of the experimental and normal (control) common carotid arteries were $1.274 \pm 0.162$ and $0.089 \pm 0.023$ (mean \pm SEM), respectively ( $p<0.001)$. Conclusion: This technique is simple, inexpensive, and highly reproducible and it induces sufficient $\mathrm{MIH}$ to study this phenomenon in animal models.

$$
\begin{aligned}
& \text { (c) } 2018 \text { The Author(s) } \\
& \text { Published by S. Karger AG, Basel }
\end{aligned}
$$

\section{Introduction}

The development of arterial myointimal hyperplasia (MIH) has a significant impact on the long-term outcomes of vascular procedures such as bypass surgery and angioplasty. Experimentally induced $\mathrm{MIH}$ in animal models is used for the study of the pathogenesis of this 
Fig. 1. a Power Flosser device. b Technique of inducing myointimal hyperplasia in the carotid artery. The dotted ties on the common carotid artery (CCA) and the internal carotid artery (ICA) are the stay sutures used to temporarily interrupt the blood flow during the procedure. The loose tie (blue) at the origin of the external carotid artery (ECA) is used to tie the artery after completion of the procedure. The rotating tip is seen within the CCA via an incision in the ECA just beyond its take off from the CCA. For better clarity in the diagram, a space is shown between the rotating tip and the wall of the CCA; in real life the device is introduced with some difficulty into the lumen of the CCA and it sits snugly within the lumen. Upon starting the machine, the floss tip is in firm contact with the intima and thus makes the required damage.

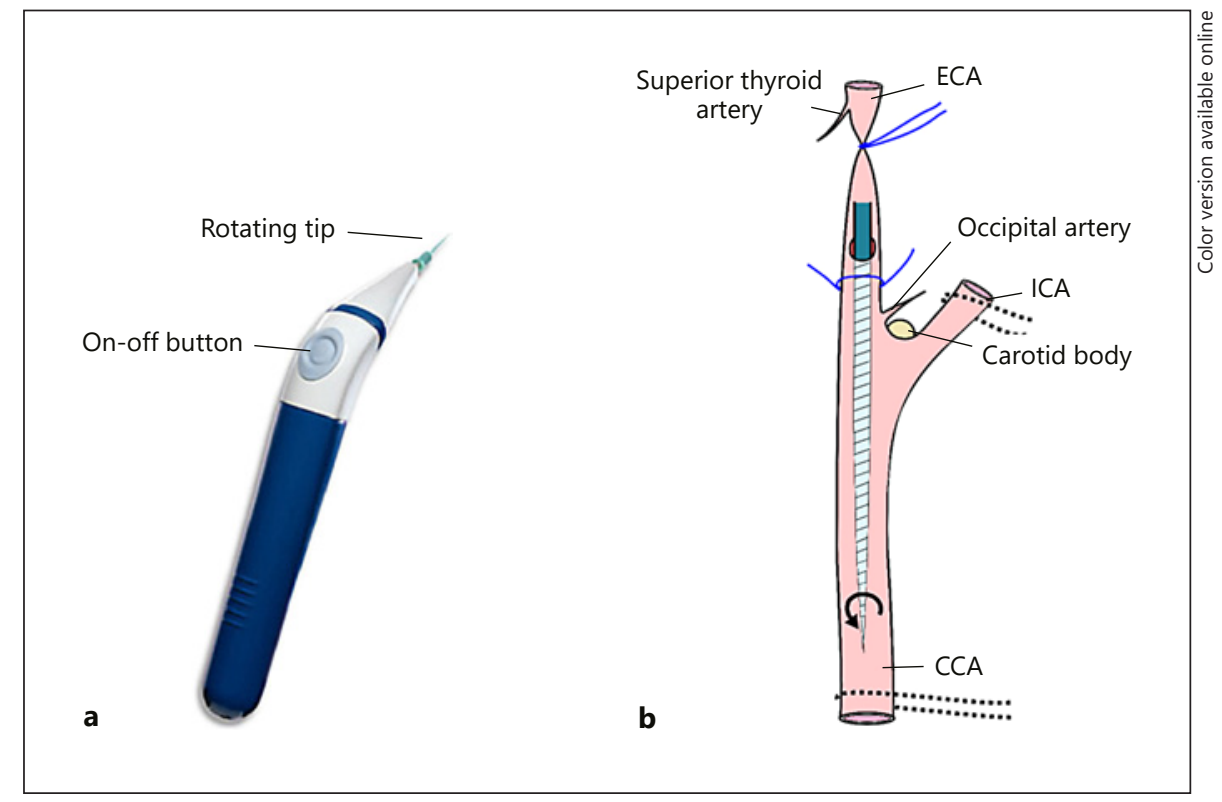

condition and to investigate ways to prevent or decrease its development. Most studies have used balloon angioplasty in animal models to induce MIH [1-3]. However, these methods are inherently tedious and require the use of one balloon catheter for each procedure, with the attached cost. In addition, these models were found not to be standardized because the pressure within the balloon may vary depending on the operator's maneuver (i.e., the balloon is not inflated equally within the same carotid, and it is not the same in different applications) [4-8]. We report a new technique to induce MIH using a dental flossing cachet, which is not expensive compared to the balloon and can be easily purchased. Unlike experimental balloon angioplasty, which entails the complete denudation of the intima from the carotid artery, this technique is automated and induces injury only to the intima by the rotating tip of the flossing cachet, which would stimulate the endothelial cells to initiate the process of intimal hyperplasia, mimicking the clinical procedures of angioplasty or bypass anastomosis.

\section{Materials and Methods}

This study was approved by the Animal Protection Ethics Committee. Ten inbred Wistar rats (body weight $\geq 400 \mathrm{~g}$ ) were maintained at the Animal Resources Centre of the Health Science Center, Kuwait University. The animals had free access to rat chow and tap water and were maintained in a standard 12-h light/dark cycle; the temperature was maintained at $25 \pm 2{ }^{\circ} \mathrm{C}$.

\section{Induction of $M I H$}

Before the operation, the rats were anesthetized by intramuscular injection of a mixture of ketamine $(85 \mathrm{mg} / \mathrm{kg})$ and rumpun $(5$ $\mathrm{mg} / \mathrm{kg}$ ) and placed supine on a sterile surgical sheet spread over an electrically warmed blanket. Using a clipper, the animal fur in front of the neck was shaved, and the skin was cleaned with betadine and alcohol. A midline neck incision was made extending from the sternal angle to the level of the larynx. Using the operating microscope, a blunt dissection was made on one side of the trachea to approach the common carotid artery (CCA) which was extended up to the angle of the mandible to expose the carotid bifurcation.

Two loose stay sutures (silk $3 / 0$ suture) were applied, i.e., one around the CCA as it entered the neck and the other on the internal carotid artery (ICA). A ligature was tied around the external carotid artery (ECA), as distal as possible towards the lower jaw. The animal was anticoagulated by intramuscular injection of heparin (50 IU $/ \mathrm{kg}$ of body weight). The 2 branches of the ECA (occipital and superior thyroid arteries) were dissected and cauterized to facilitate mobilization of the ECA and to prevent retrograde bleeding during the procedure. A loose suture was applied around the origin of the ECA which was used to ligate it at the end of the procedure.

Blood flow from the CCA and the ICA was temporarily interrupted by pulling on the loose stay suture. The ECA was then snipped with a pair of fine microscopic scissors just distal to its origin from the CCA, and then the dental flossing cachet rotating tip (WaterPik FLA-220 Power Flosser; South Shelton, CT, USA) was inserted through this opening into the ECA down to the CCA (6-8 $\mathrm{mm}$ in the distal CCA, just before its bifurcation) (Fig. 1). Once the device was in its proper position within the CCA, the button of the device was pressed to start the spiraling motion of the tip for 3 rounds of $20 \mathrm{~s}$ each to induce intimal damage in the distal CCA. The tip of the device was then withdrawn, and the loose ligature at the origin of the ECA was tied flush with the CCA bifurcation. The blood flow was resumed through the CCA and the ICA by removing the loose stay sutures. The contralateral CCA served 
Fig. 2. Rat common carotid artery crosssection stained with hematoxylin and eosin. a Experimental common carotid artery showing intimal proliferation and thickening (magnification $\times 10$ ). b Control common carotid artery indicating a normal structure of its wall (magnification $\times 10$ ). c Microphotograph of the boxed area in a showing the experimental arterial structure (internal elastic lamina, IEL; external elastic lamina, EEL). The area inside of the IEL is the intima, and the area between IEL and EEL is the media. Smooth muscle cell proliferation and migration (blue arrows) lead to neointima formation and an increase in the intima/media ratio (magnification $\times 40$ ). d Microphotograph of the boxed area in $\mathbf{b}$ showing the normal arterial structure. The area inside of the IEL is the intima, and the area between IEL and EEL is the media (magnification $\times 40$ ).
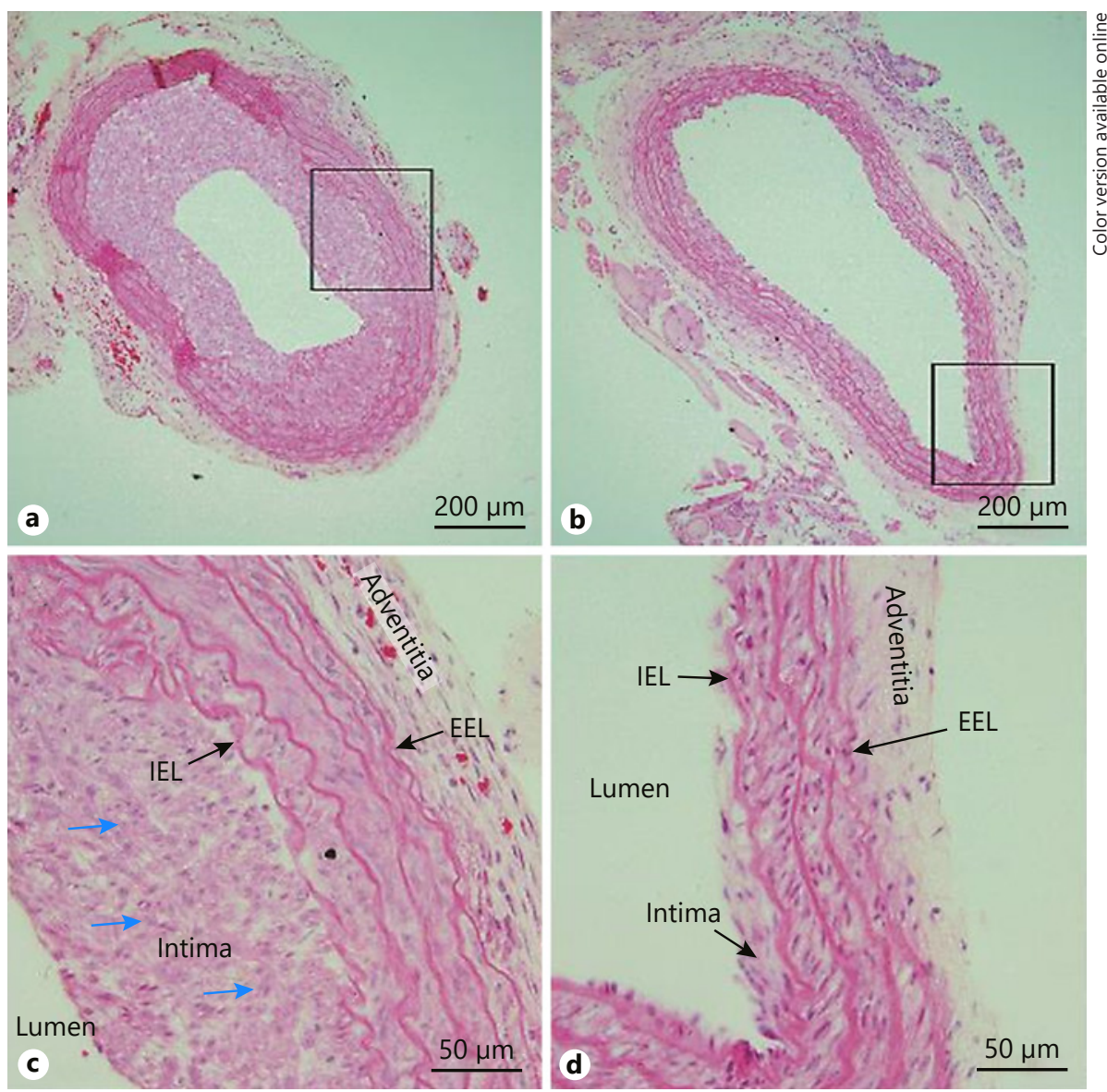

as a control by dissecting it up to the angle of the jaw and by the application of loose stay sutures at the proximal and distal ends of the artery, which were ultimately removed before wound closure. At the end of the procedure, the wound was washed with warm sterile normal saline, and the retracted muscles were returned to their normal anatomic position in the midline. The skin incision was closed with continuous $3 / 0$ silk sutures, and the animals were kept warm until recovery, transferred to a designated cage, and allowed free access to food and water.

After 2 weeks, the animals were anesthetized and the neck wounds opened. The experimental site was exposed first, and the CCA was dissected all along to its bifurcation. A stay suture was passed around the proximal CCA at the root of the neck. This suture was tied, immediately followed by injection of normal saline (using an insulin needle and syringe) into the CCA to flush out the blood. The CCA, along with its bifurcation, was then harvested and fixed in formalin. The contralateral (control) CCA was likewise flushed, harvested, and fixed. Finally, the animals were euthanized via an overdose of anesthesia. The tissue was fixed in $10 \%$ formalin and embedded in paraffin blocks, and cut and cross-sections of the harvested arteries were stained with a standard hematoxylin and eosin stain.

\section{Morphometric Analysis}

Digital images of the harvested arteries were captured using a computerized imaging system and analyzed. Morphological analysis was performed by tracing the interior surface of the lumen, the internal elastic lamina, and the external elastic lamina (EEL). The area inside of the internal elastic lamina is the intima, and the area between internal elastic lamina and external elastic lamina is the media $[9,10]$. Morphometric analysis was done by one of the authors (N.K.), who was blinded to the origin of the specimens.

\section{Statistical Analysis}

All data are presented as means \pm SEM. Comparison of histological findings between the control and experimental CCA groups was made via an unpaired Student $t$ test. $p<0.05$ was considered statistically significant. All statistical analyses were performed with GraphPad Prism version 6.0 (GraphPad Software Inc., San Diego, CA, USA).

\section{Results}

All rats appeared healthy and were moving all limbs before sacrifice, and no adverse effects were observed. All of the neck incisions healed well, with no infections observed. 


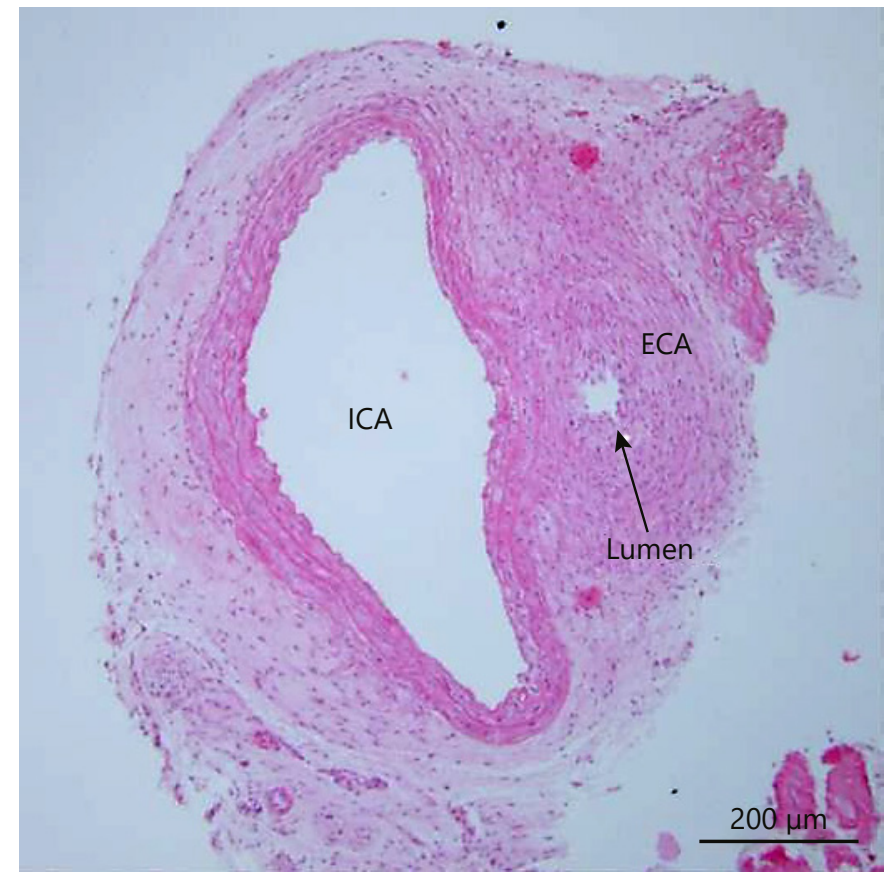

Fig. 3. Rat carotid artery cross-section at the bifurcation of both internal (ICA) and external (ECA) carotid arteries stained with hematoxylin and eosin. The ECA shows near complete luminal occlusion due to the myointimal hyperplasia (magnification $\times 10$ ).

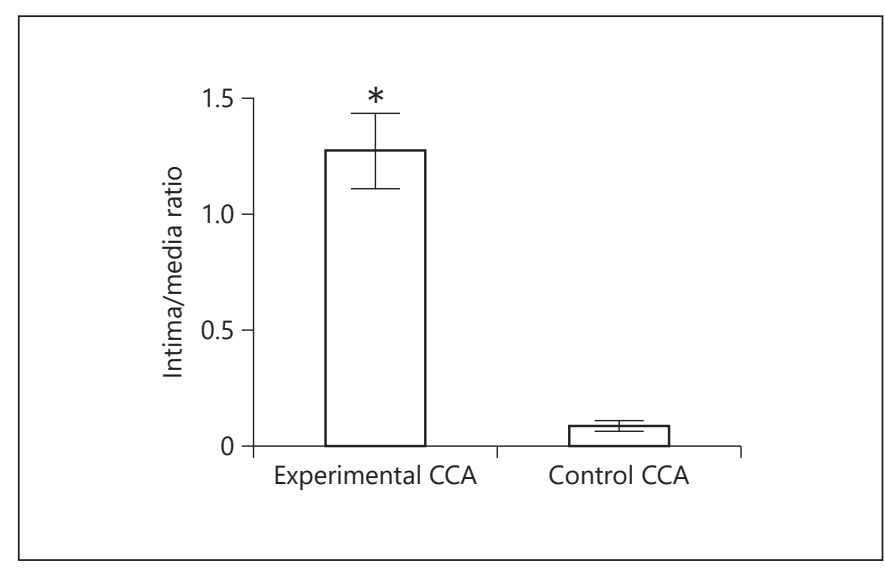

Fig. 4. Comparison of intima/media ratios in experimental vs. control common carotid arteries (CCA) ( $n=10$ for each group). ${ }^{*}$ The ratio was significantly higher $(p<0.001)$ in the experimental group $(1.274 \pm 0.162)$ compared to the control group $(0.089 \pm 0.023)$.

The cross-section of the experimental carotid arteries (Fig. 2a, c) shows impressive intimal proliferation and thickening compared to the control carotid arteries (Fig. 2b, d). The MIH was noted to extend from the site of the insertion of the flosser's tip in the ECA (Fig. 3) down to the whole length of the CCA, i.e., the site of intimal contact with the rotating tip of the flosser. Figure 3 shows the bifurcation of the CCA, in which the ECA lumen is nearly completely occluded due to the MIH. Figure 4 shows that an extensive MIH was induced in the experimental carotid arteries as indicated by the higher intima/media ratios $(1.274 \pm 0.162)$ compared to the control common carotid arteries $(0.089 \pm 0.023)$ (mean \pm SEM); this difference was highly significant $(p<0.001)$.

\section{Discussion and Conclusion}

Experimental MIH has been induced previously in rat carotid arteries using a balloon catheter; however, this technique does not mimic the real-world situation because it denudes the whole intimal layer, which does not occur during clinical angioplasty [1-3]. Moreover, the small Fogarty (balloon) catheter is used only once per animal and it is therefore expensive. We opted to explore the use of a new method to induce MIH in the rat carotids, which would mimic the clinical situation (i.e., injury to the arterial intima) rather than denuding the whole intima.

In our study, the automated flossing tip induced impressive intimal proliferation and thickening compared to the control. To prove the reproducibility and validity of the technique, the procedure was performed on 10 rats by 2 coauthors (A.S. and F.A.-O.). In all animals, $\mathrm{MIH}$ was consistently induced without variability along the CCA. In comparison, many reports have shown that the balloon injury model in rats is not standardized because the value of the pressure in the balloon is not automated and may vary depending on the operator's maneuver [4-8]. Thus, our model of intimal injury is simple, inexpensive, and highly reproducible and induces sufficient MIH to study this phenomenon in the animal model.

\section{Acknowledgments}

The authors are grateful to S. Varghese for the technical work and to the Animal Resources Center for maintaining the experimental animals. 


\section{References}

1 Chen SJ, Li H, Durand J, et al: Estrogen reduces myointimal proliferation after balloon injury of rat carotid artery. Circulation 1996; 93:577-584.

2 Clozel JP, Müller RK, Roux S, et al: Influence of the status of the renin-angiotensin system on the effect of cilazapril on neointima formation after vascular injury in rats. Circulation 1993;88:1222-1227.

3 Fingerle J, Au YP, Clowes AW, et al: Intimal lesion formation in rat carotid arteries after endothelial denudation in absence of medial injury. Arteriosclerosis 1990;10:1082-1087.
4 Clowes AW, Reidy MA, Clowes MM: Kinetics of cellular proliferation after arterial injury. 1 . Smooth muscle growth in the absence of endothelium. Lab Invest 1983;49:327-333.

5 Clowes AW, Schwartz SM: Significance of quiescent smooth muscle migration in the injured rat carotid artery. Circ Res 1985;56:139145.

6 Indolfi C, Esposito G, Di Lorenzo E, et al: Smooth muscle cell proliferation is proportional to the degree of balloon injury in a rat model of angioplasty. Circulation 1995;92: 1230-1235.

7 Tulis DA: Rat carotid artery balloon injury model. Methods Mol Med 2007;139:1-30.
8 Zhang W, Trebak M: Vascular balloon injury and intraluminal administration in rat carotid artery. J Vis Exp 2014;e52045.

9 Krueger KD, Mitra AK, DelCore MG, et al: A comparison of stent-induced stenosis in coronary and peripheral arteries. J Clin Pathol 2006;59:575-579.

10 Ward MR, Kanellakis P, Ramsey D, et al: Eplerenone suppresses constrictive remodeling and collagen accumulation after angioplasty in porcine coronary arteries. Circulation 2001;104:467-472. 